\title{
P157: Face-touching: a frequent habit for self-inoculation of transmissible infections?
}

\author{
A Kwok ${ }^{*}$ M-L McLaws \\ From 2nd International Conference on Prevention and Infection Control (ICPIC 2013) \\ Geneva, Switzerland. 25-28 June 2013
}

\section{Introduction}

Touching the mucous membranes of the nose and mouth is a potential for transmission and acquisition of a range of infections. Infection may be spread to others after inoculating ones own hands during face-touching or infections may be acquired via contaminated hands after face-touching.

\section{Objectives}

To investigate the prevalence of face-touching behaviour in medical students.

\section{Methods}

All fifth year UNSW medical students who had completed 4 hours of infection control education in the prior year attending a lecture theatre for a $60 \mathrm{~min}$ lecture were invited to participate in a video recording for a behaviour observational study. To eliminate bias students were blinded from the aim of the study. Consented students were instructed to sit on one side of the lecture theatre where video recorder was set up. University Ethics approval was obtained. Two researchers observed video tapes independently and tallied the frequency of hand-to-face contacts for each participant using a standardised sheet to record the region (nose, mouth, eye and non-mucous membrane regions) and frequency of each of these regions.

\section{Results}

All 29 students touched their face at least once. 90\% $(26 / 29)$ touched a mucous membrane on the face at least once during $60 \mathrm{~min}$ of observation. Out of the 2346 touches observed, 1175 were to a non-mucous membrane region and 1171 were touches to nose, mouth or eye regions, with an average 45 mucous membrane touches per student over the 60 mins period (median 29, min 4, max 153). Touching the mouth was the most frequent region at 372 touches, followed by the nose 318 touches and eyes 273 touches. The duration for mouth touching ranged from $1 \mathrm{sec}$ to $12 \mathrm{sec}$ (median $1 \mathrm{sec}$, mean $2 \mathrm{sec}$ ), the duration for nose touching ranged from $1 \mathrm{sec}$ to $10 \mathrm{sec}$ (median $<1 \mathrm{sec}$, mean $1 \mathrm{sec}$ ), eye touching ranged from 1 sec to 5 sec (median $<1 \mathrm{sec}$, mean $1 \mathrm{sec}$ ).

\section{Conclusion}

The greatest shedding of virus in the community occurs during the prodromal stage of influenza usually 3 days before symptoms and signs. During the prodromal period the prevalent behaviour of face-touching provides the opportunity for acquisition and transmission of infectious material. During public health campaigns to educate and alter the community about reducing their risk of transmission and acquisition the campaign should also focus on modifying our unconscious preening behaviour.

\section{Disclosure of interest}

None declared.

Published: 20 June 2013

doi:10.1186/2047-2994-2-S1-P157

Cite this article as: Kwok and McLaws: P157: Face-touching: a frequent

habit for self-inoculation of transmissible infections? Antimicrobial

Resistance and Infection Control 2013 2(Suppl 1):P157.

UNSW Medicine, The University of New South Wales, Sydney, Australia

(c) 2013 Kwok and McLaws; licensee BioMed Central Ltd. This is an Open Access article distributed under the terms of the Creative 